\title{
Effect of Oral Aspirin on Plasma Levels of Vitamin K-Dependent Clotting Factors - Studies in Healthy Volunteers
}

\section{Dear Sir,}

The rationale for the use of aspirin as an antithrombotic drug lies mainly in its inhibition of thromboxane $\mathrm{A}_{2}$-dependent platelet function (1). There is also clinical (2) and experimental evidence (3) that in some instances aspirin is antithrombotic at doses higher than those required to inhibit the platelet cyclooxygenase, suggesting that at high doses aspirin may also act through mechanism(s) other than its effects on platelet function. Among the mechanisms by which aspirin might be antithrombotic is inhibition of blood coagulation (3). Case reports of patients treated with aspirin for rheumatoid arthritis $(4,5)$ and non-controlled studies on healthy volunteers $(5,6)$ have shown that massive doses of aspirin (3.7-10 g/day, orally, or 2-20 g/day, i.v.) prolong the prothrombin time by depressing the plasma levels of the vitamin K-dependent clotting factors. However, there has been no controlled study of the effects on the coagulation system of aspirin given orally at high dose (ca. $1.3 \mathrm{~g} / \mathrm{day}$ ) proven to be antithrombotic in some clinical trials (2).

In a double blind, placebo-controlled, cross-over study we evaluated the effects of 8 days of oral administration of aspirin $(20 \mathrm{mg} / \mathrm{kg}$ divided into three daily doses) to 14 healthy volunteers ( 7 men, 7 women, aged 22-37) on the prothrombin time, partial thromboplastin time and on the plasma levels of vitamin $\mathrm{K}$ dependent factors II, VII, IX, X, protein C, and factor V (taken as an example of non vitamin $\mathrm{K}$-dependent clotting factors). The plasma levels of clotting factors were tested with biological assays (7) in blind by two different technicians on days 1 (before the first dose), 2, 4, 6, 8 and 10 (two days after discontinuation of the drug). The data reported by each technician were averaged. Aspirin caused a slight, but statistically significant decrease of factor II $(\mathrm{p}<0.05)$, which returned to basal values after discontinuation of the drug (Table 1). Aspirin did not affect the plasma levels of the other clotting factors studied (Table 1), nor of prothrombin time and partial thromboplastin time (not shown). No statistically significant difference between men and women

Table 1 Effects in 14 healthy volunteers of oral administration of aspirin $\left(20 \mathrm{mg} \mathrm{kg}^{-1} \mathrm{day}^{-1}\right)$ on plasma levels of vitamin K-dependent clotting factors, protein $\mathrm{C}$ and factor $\mathrm{V}$

\begin{tabular}{lrcccr}
\hline \multirow{2}{*}{$\begin{array}{l}\text { Clotting } \\
\text { factor }\end{array}$} & \multicolumn{2}{c}{ Percent of basal value } & & \\
Day 2 & Day 4 & Day 6 & Day 8 & \multicolumn{1}{c}{ Day 10 } \\
\hline II* & $94 \pm 3$ & $90 \pm 4$ & $90 \pm 3$ & $90 \pm 4$ & $98 \pm 4$ \\
VII & $99 \pm 4$ & $95 \pm 4$ & $96 \pm 4$ & $100 \pm 4$ & $103 \pm 3$ \\
IX & $100 \pm 7$ & $93 \pm 4$ & $101 \pm 7$ & $100 \pm 10$ & $100 \pm 6$ \\
X & $95 \pm 3$ & $95 \pm 4$ & $94 \pm 4$ & $96 \pm 6$ & $103 \pm 4$ \\
Protein C & $93 \pm 2$ & $95 \pm 2$ & $95 \pm 3$ & $99 \pm 4$ & $99 \pm 3$ \\
V & $97 \pm 3$ & $98 \pm 2$ & $99 \pm 3$ & $98 \pm 3$ & $99 \pm 5$ \\
\hline
\end{tabular}

Mean \pm S.E.M.

* Values for days 2, 4, 6 and 8 were significantly different from $100 \%$ ( $p<0.05$, analysis of variance).

Plasma salicylate levels ranged between 2.6 and $7.6 \mathrm{mg} / 100 \mathrm{ml}(8)$ was found. No significant change was observed during placebo administration.

The data of this double blind, placebo-controlled, cross-over study suggest that, at the daily dose of $20 \mathrm{mg} / \mathrm{kg}$, aspirin does not affect the synthesis of the vitamin K-dependent clotting factors (except perhaps for a mild reduction of factor II) or of protein C. They are in contrast with those of previous non-controlled studies, in which prolongation of the prothrombin time (5) and a decrease in vitamin $\mathrm{K}$-dependent clotting factors to about $70-80 \%$ of basal values (6) were seen. Differences in the dose of aspirin between the previous studies (10 g/day orally or 2 g/day i. v.) and the present study (ca. $1.3 \mathrm{~g} /$ day orally) might account for the different results obtained.

It is unlikely that aspirin, given at the dose and for the period of time tested in this study would have antithrombotic effects mediated by inhibition of coagulation, although the mild inhibitory effect on factor II observed in the present study might synergize with the inhibition of the platelet cyclooxygenase in mediating the antithrombotic action of aspirin.
M. Cattaneo
A. D'Angelo
M. T. Canciani
D. Asti
S. Viganò-D'Angelo
A. Tripodi
P. M. Mannucci

A. Bianchi Bonomi Hemophilia and Thrombosis Ctr.

Maggiore Hospital and University of Milano

Milano, Italy

\section{References}

1 Packham M A, Mustard J F. Pharmacology of platelet-affecting drugs. Circulation 1980; 62 (suppl. V): V26-V41.

2 Marcus A J. Aspirin as an antithrombotic medication. N Engl J Med 1983; 309: 1515-7.

3 Cattaneo M, Chahil A, Somers D et al. Effect of aspirin and sodium salicylate on thrombosis, fibrinolysis, prothrombin time, and platelet survival in rabbits with indwelling aortic catheters. Blood 1983; 61: 353-61.

4 Clausen H G, Jager B V. The relation of the plasma salicylate level to the degree of hypoprothrombinemia. J Lab Clin Med 1946; 3: 428-35.

5 Goldsweig H G, Kapusta M, Schwartz J. Bleeding, salicylates and prolonged prothrombin time: three case reports and a review of the literature. J Rheumatol 1976; 3: 37-42.

6 Loew D, Vinazzer H. Dose-dependent influence of acetylsalicylic acid on platelet functions and plasmatic coagulation factors. Haemostasis 1976; 5: 239-49.

7 Ingram G I C, Brozovic M, Slater N G P. Bleeding disorders. Investigation and Management. Blackwell Scientific Publications 1982.

8 Trinder P. Rapid determination of salicylate in biological fluids. Biochem J 1954; 57: 301-3.

Received December 24, 1987 Accepted December 29, 1987 